\title{
Measuring and Estimating the Propagation Path Loss and Shadowing Effects for Marine Wireless Sensor Networks at 5.8 GHZ
}

\author{
J. C. Reyes-Guerrero, Member, IEEE, Gordan Sisul, Member, IEEE, and Luis A. Mariscal, Member, \\ IEEE
}

\begin{abstract}
This work presents experimental propagation measurements over sea at $5.8 \mathrm{GHz}$ for a radio link between an antenna installed aboard a boat and an antenna installed onshore. Experimental data include path loss and large-scale fading. We focus on modeling obstructed paths. Measured local mean power values show that a multi-slope path loss model are more appropriate than traditional models that use only one slope for NLOS (Non-Line-of-Sight) transmissions. The received signal was found to attenuate at the rate of up to $130 \mathrm{~dB}$ per decade in some shadow regions. It was also found that measurements fit reasonably well the two-ray model while LOS (Line-Of-Sight) condition remains. This investigation is useful, among others, for planning Wireless Sensor Networks offshore.
\end{abstract}

Keywords - Large-scale path loss measurements, sea WiMAX, Wireless Sensor Networks, 5.8 GHz.

\section{INTRODUCTION}

$\mathrm{T}$ HE current wireless technologies used in marine applications are mainly based on VHF, cellular mobile telecommunication systems (GSM, UMTS, etc.) and satellite communication systems (INMARSAT, VSAT, etc.). However, these systems suffer from lots of weaknesses like low bandwidth and capacity (GSM, Satellite and VHF systems), short range (cellular mobile telecommunication systems), high cost for certain applications (satellite and cellular mobile telecommunication systems) and large size and weight of antennas and hardware transceivers (VHF systems) [1]. These limitations have motivated a new research activity aiming to design and develop a novel broadband wireless communication system to perform applications like those related to real-time monitoring of the marine environment through sensing multiple physical parameters.

A Wireless Sensor Network (WSN) [2] based on

José Carlos Reyes-Guerrero is with the University College of Marine, Nautical and Radio-Electronic Engineering, University of Cadiz, Av. Republica Saharaui sn, 11510 Puerto Real, Spain (phone: 34 956016265; e-mail: josecarlos.reyes@uca.es).

Gordan Sisul is with the Faculty of Electrical Engineering, University of Zagreb, Unska 3, 10000 Zagreb, Croatia; (phone: 385-1-6129742, e-mail: gordan.sisul@fer.hr).

Luis Antonio Mariscal-Rico is with the University College of Marine, Nautical and Radio-Electronic Engineering, University of Cadiz, Av. Republica Saharaui sn, 11510 Puerto Real, Spain (phone: 34956016493; e-mail: luis.mariscal@uca.es).
WiMAX standard ([3]-[5]) could be a good candidate to accomplish this task. WSNs offer a new paradigm for marine monitoring. They are a type of autonomous, selforganized ad-hoc network composed of tens, hundreds or even thousands of smart sensor nodes that can monitor large physical environments. In a WSN, sensor nodes have not only the sensor component, but also on-board processing, communication, power, and storage capabilities. All of them are limited resources. A general architecture of this kind of network is showed in Fig. 1. It is composed of sensor nodes which could be generally buoys, ships or stable platforms over sea which, in their turn, transmit the data wirelessly to a sink node by using multihop communications; i.e., a node could send the measured data to the base station through intermediate nodes. The sink node is in charge of gathering all data and transmitting them to a base station on land for processing and monitoring purposes. The wireless link between the sink node and the base station is critical for the marine WSN. This is why we focus on this kind of links in this work.

WiMAX is an evolving technology optimized to operate on land environments where its good performance has been extensively demonstrated. Several frequency bands can be used for deploying this system. The license-exempt $5 \mathrm{GHz}$ band is of interest to WiMAX, because it is generally available worldwide and free for anyone to use, i.e., it could enable deployments in underserved markets

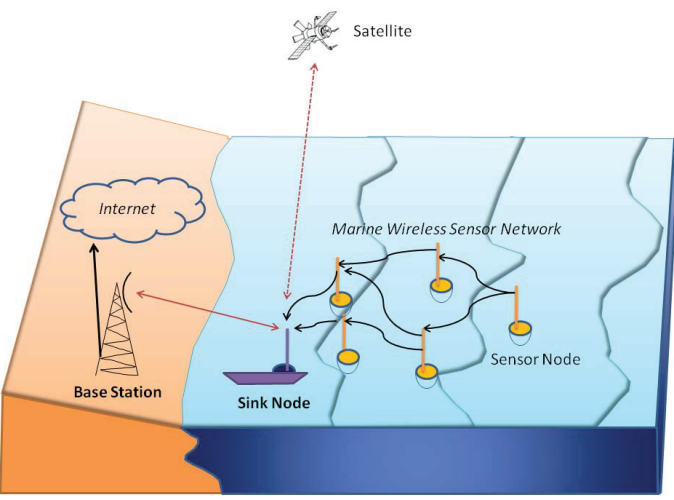

Fig. 1. General architecture of a marine Wireless Sensor Network 
like the maritime ones. In particular, it is the upper 5.725 $\mathrm{GHz}-5.850 \mathrm{GHz}$ band that is most attractive due to the fact that many countries allow higher power output compared to other bands. This facilitates less costly deployments. Regarding range and peak data rates, field tests, on land, have shown tens of kilometers and Mbps, respectively. All these potential characteristics overcome the weaknesses described above. However, the performance of WiMAX networks in marine environments is not optimum due to the different radio propagation conditions. Hence, the main goal is to optimize the WiMAX standard for maritime applications.

As a first step, knowledge of $5 \mathrm{GHz}$ radio propagation over sea is required. Propagation measurements for land have been discussed extensively [6]. Further works in this field have been done in urban and suburban environments [7], [8]. In maritime wireless links, large-scale experimental propagation characteristics, for different radio conditions and configurations, were shown in previous studies [9]-[13]. However, shadowing effects due to buildings, large infrastructures and boats have not been investigated in depth. In this work, averaged received power values are shown along a route planned to determine the propagation characteristics to shadow sensor nodes.

\section{Measurement EnVIRONMENT AND Route}

Cadiz bay (Spain) was selected to represent a maritime challenge scenario where it is possible to take into account a lot of environment characteristics. This zone has a heterogeneous topography with dense populated urban areas including large infrastructures and buildings. Moreover, some nautical clubs and an important commercial port are placed along the shore. Therefore, large and small ships are anchored around and the fairways are very dynamics.

The measurements were carried out in a sunny day. The temperature ranged between 21 and $25.6{ }^{\circ} \mathrm{C}$. The humidity was around $95 \%$. The sea condition was calm and there were no large waves. The atmospheric pressure was about $1009 \mathrm{hPa}$. The wind speed reached $12.5 \mathrm{~m} / \mathrm{s}$.

In order to investigate the impact of this kind of environments on the transmitted signal, a measurement campaign was planned over a route. Fig. 2 shows the fixed location of the receiver antenna (RX) installed onshore and the route followed by the ship (from A to F). The ship was moving along the route at about $2.5 \mathrm{~m} / \mathrm{s}$. Some waypoints are depicted in Fig. 2 in order to explain some important shadowing effects.

Strictly speaking, it is difficult to maintain LOS (LineOf-Sight) conditions in real marine scenarios due to temporal LOS blocking by ships and sea waves. Besides, the curvature of the earth blocks the signal gradually with the distance. In Fig. 2, measurements were carried out in LOS conditions from A to $\mathrm{B}$. The signal was partly blocked from B to $\mathrm{C}$, and in this way a ship approached and blocked the signal for a few seconds. In the way from $\mathrm{C}$ and D the LOS signal was totally blocked by ships and

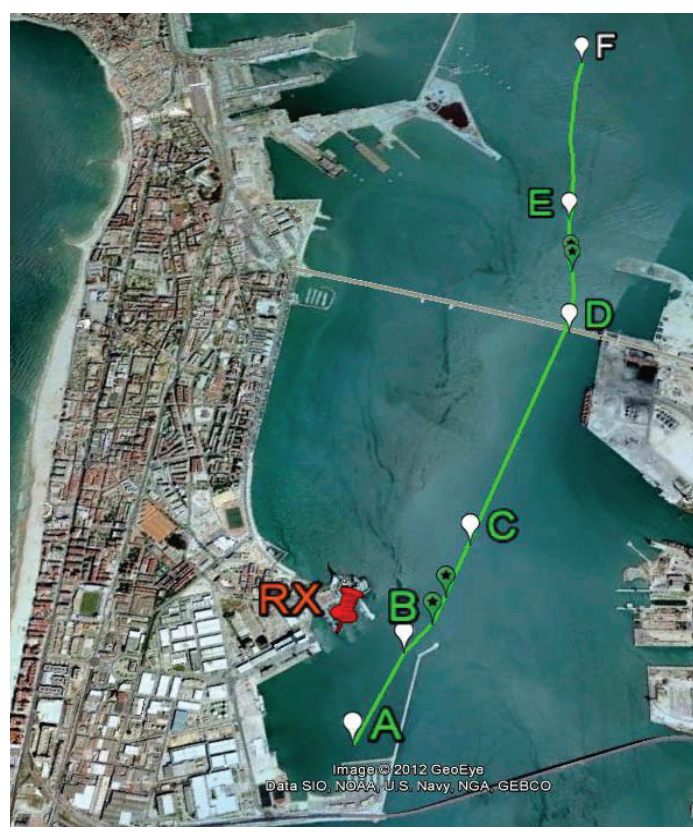

Fig. 2. Fixed location of the receiver antenna onshore (RX) and route followed by the ship (from A to F) during the measurement campaign.

buildings near the receiver. Furthermore, a large infrastructure (a bridge) influenced the transmission from $\mathrm{D}$ to $\mathrm{F}$. Also, in this last way, some boats blocked the direct path.

\section{Measurement System And Signal Processing}

Measurements were carried out by transmitting a 30 $\mathrm{dBm}$ Continuous Wave (CW) from an antenna installed on a ship and receiving this signal in a receiver installed aboard a ship onshore. This measurement system is fully described in Fig. 1. A $0 \mathrm{dBm} \mathrm{CW}$ signal at $5.8 \mathrm{GHz}$ was transmitted from a signal generator. This output $\mathrm{CW}$ signal was further amplified $33 \mathrm{~dB}$ by a broadband amplifier up to $1 \mathrm{~W}$ (transmitter cable loss was $3 \mathrm{~dB}$ ). This signal was the input of the antenna installed on the ship. The used antenna had the following main characteristics: $9 \mathrm{dBi}$ gain, vertical polarization, omnidirectional radiation pattern in the horizontal plane and $7^{\circ}$ beam-width in the vertical plane. This transmitter antenna was mounted $2.7 \mathrm{~m}$ from the sea surface. The receiver antenna was the same as the transmitter one and it was mounted $5.45 \mathrm{~m}$ from the sea surface. The received signal by the antenna was the input to a vector signal analyzer which was in charge of measuring it. A computer was connected to the analyzer in order to record the received signal to further processing. In addition, a GPS (Global Position System) device was also

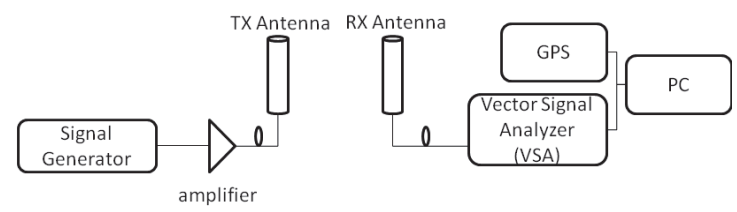

Fig. 3. Block diagram of the measurement system. 
connected to the computer to time-stamp the received signal. Moreover, the GPS signal acquired by the computer was useful to calculate the separation distance between the transmitter and the receiver.

The instantaneous received signal from a CW transmission is measured. Therefore, shadowing and multipath effects are present in the measurements. We need to separate both variations in order to perform an independent study. The separation is performed by means of a temporal window that is slid through the data and calculates the local mean power every second. Due to ship natural movements on the sea and speed, the mean operation was made over a distance of several wavelengths.

\section{RESULTS}

All measurements carried out as explained in Section III over the planned route are shown in Fig. 4. Moreover, the results expected from the two-ray model for vertical polarization transmission are also shown as a reference [12]. The differences between the measurement results and the results expected by the theoretical model can be explained according to the propagation environment.

Signal dispersions on the received signal are partly due to antenna movement effects and characteristics; this should be taken into account to compare experimental data with theoretical models which assume isotropic antennas. Despite of this, measurements fit reasonably well theoretical results in the route from $\mathrm{A}$ to $\mathrm{B}$, where the measurements were carried out in LOS conditions. However, measurements were carried out in NLOS conditions from $\mathrm{B}$ to $\mathrm{F}$, as it was checked experimentally.

In order to accurately describe our propagation scenario for NLOS conditions, an empirical path loss model is developed using experimental data over the route from B to F. One of the simplest and most common models used to predict the expected average level of received power for a given transmitter-receiver separation, $P_{R X}(d)$, is indicated in (1):

$$
P_{R X}=P_{R X}\left(d_{0}\right)-10 n \log _{10}\left(\frac{d}{d_{0}}\right)-\chi \text {, for } d>d_{0}
$$

which groups all various effects into two parameters: the path loss exponent $n$, and the zero-mean Gaussian random

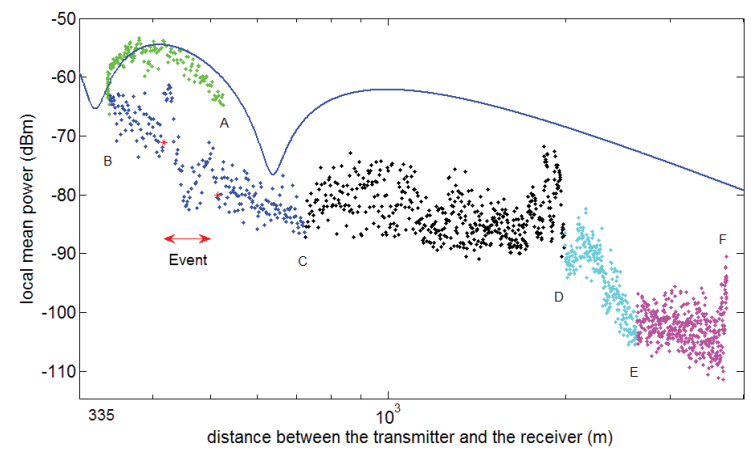

Fig. 4. All local mean power values measured along the planned route. The two-ray model for vertical polarization transmission is also shown.

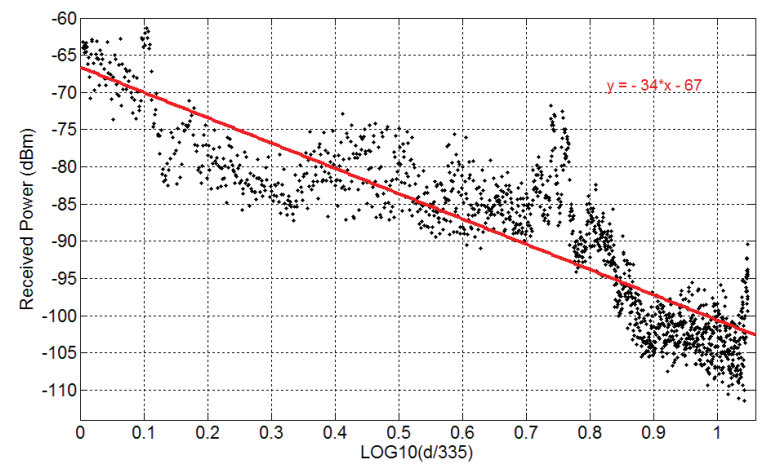

Fig. 5. NLOS measurements and linear regression analysis.

variation $\chi$, that represents the shadow fading effect. In this model, $P_{R X}\left(d_{0}\right)$ is the expected level of received power at a reference distance $d_{0}$ and it should be determined from measurements.

The shadowing value $X$ is typically modeled as a normal random variable, i.e.:

$$
\mathrm{X} \sim N\left(0, \sigma^{2}\right)
$$

where $N\left(0, \sigma^{2}\right)$ is a Gaussian (normal) distribution with mean 0 and standard deviation $\sigma$, in decibel units.

Path loss models, as the represented by (1), attempt to account for the distance-dependent relationship between transmitted and received power as well as for large-scale variations in signal strength due to macroscopic objects within the propagation environment that creates shadow zones. For instance, in sea environments, large ships, buoys, coast relief, buildings near harbor, islands situated between transmitter and receiver, etc., can create high variations in the received signal.

Based on (1), linear regression analysis with minimum mean square error (MMSE) can be applied to find the path loss exponent $n$ and the standard deviation $\sigma$ for NLOS measurements. These both parameters characterize the large-scale effects in our experimental scenario. In data processing, $d_{0}$ was chosen to be $335 \mathrm{~m}$. The scatter plot is shown in Fig. 5, and the values for the path loss exponent and standard deviation were found to be 3.3975 and 5.3215 , respectively.

However, it should be noticed that the path loss exponent cannot be considered constant along the route. In fact, according to the environment and NLOS measurements in Fig. 4, path loss depends not only on distance but also on scatters in the environment. In fact, we can distinguish four different regions in which this parameter could be considered constant approximately. Therefore, the same analysis was performed separately in each zone in order to find both, the path loss exponent and the standard deviation values.

All results are summarized in Table I, where channel parameters are shown for each region. Smaller standard deviation values than in the general model were obtained. Therefore, a muli-slope model is more appropriate. Although a path loss exponent between 2 and 4 is expected by the two-ray model for the locations where the 
measurements were performed, path loss exponent values of 6.5 and 13 were found on region 1 and 3, respectively; indicating higher rate signal attenuation in a real scenario. On the other hand, for measurements on regions 2 and 4 smaller path loss exponent values that the predicted one by the free space model were found; however, higher standard deviation values were obtained for these both ensembles.

TABLE 1: RESULTS OF THE MMSE LINEAR REGRESSION FOR MEASUREMENTS IN DifFERENT REGIONS

\begin{tabular}{|l|c|c|}
\hline Region & $\boldsymbol{n}$ & $\boldsymbol{\sigma}(\boldsymbol{d B})$ \\
\hline 1 (from B to C) & 6.4618 & 3.2945 \\
\hline 2 (from C to D) & 0.7601 & 3.9514 \\
\hline 3 (from D to E) & 12.9843 & 3.0889 \\
\hline 4 (from E to F) & 0.5054 & 3.3634 \\
\hline
\end{tabular}

In Fig. 6, measurements and linear regression analysis for region 1 are depicted. A shadowing effect due to another ship can be clearly observed. The ship was moving and crossed the LOS path between the transmitter and the receiver for a few seconds. It caused a variation in signal envelope of $21 \mathrm{~dB}$.

\section{CONCLUSION}

We presented experimental results aiming to characterize wireless propagation channels over sea at 5.8 $\mathrm{GHz}$ in different shadowing situations.

Although we focus on NLOS measurements, it should be noticed that two-ray model fits measured large scale path loss reasonably well when LOS condition remains. However, when several obstacles, e.g. buildings, large infrastructures and ships, block the transmitted signal, the received signal is found to attenuate at a higher rate. A multi-slope path loss model is proposed. Specifically, the path loss exponent was found to reach a value of 13 in a

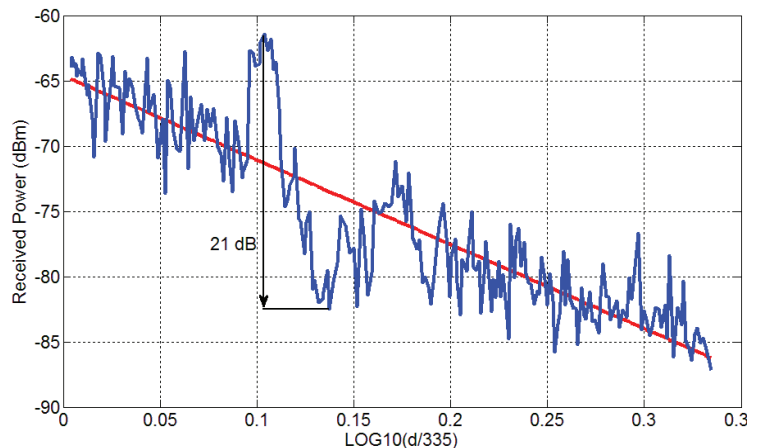

Fig. 6. Measurements and linear regression analysis for region 1. A shadow effect due to a ship can be observed. region affected by a large bridge. Also, it was shown a shadowing effect due to a moving boat that crossed the LOS path that caused a signal variation of $21 \mathrm{~dB}$.

It was also found that although for some regions the received signal attenuates at a rate smaller than the predicted one by the free space model, higher values of the standard deviation were found. This is likely due to severe absorption, diffraction and scattering mechanisms. All these effects can limit the coverage zone of WiMAX networks. Therefore, careful networking planning is needed in order to ensure broadband connectivity everywhere over sea.

\section{ACKNOWLEDGMENT}

The authors would like to thank to Instituto Hidrográfico de la Marina (Spain), Agilent Technologies and all professionals involved for their help and support in the experimental campaigns. This research work is supported by AECID (Agencia Española de Cooperación Internacional para el Desarrollo).

\section{REFERENCES}

[1] F. Bekkadal and Kun Yang, "Novel maritime mommunication technologies," Microwave Symposium MMS 2010, 25-27 August, 2010, pp. 338-341.

[2] W. Dargie and C. Poellabauer, "Fundamentals of Wireless Sensor Networks". Wiley (2010).

[3] IEEE standard 802.16d-2004

[4] IEEE standard 802.16e-2005.

[5] J.G. Andrews, A. Ghosh and R. Muhamed, "Fundamentals of WiMAX. Understanding broadband wireless networking," Prentice Hall, 2007.

[6] T.S. Rappaport, "Wireless communications principles and practice," Pretince Hall PTR, Upper Saddle River, 2002.

[7] V. Ercerg, et al., "Channel models for fixed wireless applicatios," IEEE 802.16a-03/01R

[8] V. Erceg et al., "An empirically based path loss model for wireles channels in suburban environments," IEEE Journal on Selected Areas in Communications, vol 17, No 7, July 1999, pp. 1205-1211.

[9] J. Joe et al., "Path loss measurements in sea port for WiMAX," Wireless Communications and Networking Conference, IEEE WCNC, 11-15 March, 2007, pp. 1871-1876.

[10] Rosario G. Garroppo, Stefano Giordano, Davide lacono, Alessandro Cignoni, Matteo Falzarano, "WiMAX testbed for intenrconnection of mobile navy units in operational scenarios," Military Communications Conference, IEEE MILCOM, 16-19 November, 2008, pp. 1-7.

[11] Le Roux Y.M., Menard J., Toquin C., Jolivet J.P., Nicolas F., "Experimental measurements of propagation characteristics for maritime radio links," 9th International Conference on Intelligent Transport Systems Telecommunications (ITST), 20-22 October, 2009, pp. 364-369.

[12] J.C. Reyes-Guerrero, Luis A. Mariscal, M. Bruno and A. Medouri, "Buoy-to-ship experimental measurements over sea at $5.8 \mathrm{GHz}$ near urban environments," Mediterranean Microwave Symposium, MMS2011, 8-10 September, 2011, pp. 320-324.

[13] J.C. Reyes-Guerrero, Luis A. Mariscal, M. Bruno and A. Medouri, "Wireless communications for marine sensor networks," Fourth International Workshop on Marine Technology (MARTECH 2011 WORKSHOP), 22-23 September, 2011. 